\title{
UAE-HPLC-UV: New Contribution for Fast Determination of Total Isothiocyanates in Brassicaceae Vegetables
}

\author{
Cecilia M. Fusari, ${ }^{1,2}$ Daniela A. Locatelli,, ${ }^{1,2}$ Jorgelina C. Altamirano, \\ and Alejandra B. Camargo ${ }^{1,2,4}$ \\ ${ }^{1}$ Instituto de Biología Agrícola de Mendoza (IBAM), CONICET, Almirante Brown 500, Chacras de Coria, 5505 Mendoza, Argentina \\ ${ }^{2}$ Facultad de Ciencias Agrarias, Universidad Nacional de Cuyo, Almirante Brown 500, Chacras de Coria, 5505 Mendoza, Argentina \\ ${ }^{3}$ Laboratorio de Química Ambiental, Instituto Argentino de Nivología, Glaciología y Ciencias Ambientales (IANIGLA), \\ CONICET, Avenida Ruiz Leal s/n, Parque General San Martín, 5500 Mendoza, Argentina \\ ${ }^{4}$ Facultad de Ciencias Exactas y Naturales, Universidad Nacional de Cuyo, Padre Jorge Contreras 1300, \\ Parque General San Martín, 5500 Mendoza, Argentina
}

Correspondence should be addressed to Alejandra B. Camargo; alebcamargo@gmail.com

Received 26 August 2015; Revised 11 November 2015; Accepted 19 November 2015

Academic Editor: Patricia Valentao

Copyright (C) 2015 Cecilia M. Fusari et al. This is an open access article distributed under the Creative Commons Attribution License, which permits unrestricted use, distribution, and reproduction in any medium, provided the original work is properly cited.

\begin{abstract}
Total isothiocyanates content (ITC) is considered a good indicator of bioactive compounds responsible for beneficial effects related to Brassicaceae vegetables. Analytical performance is a critical factor for routine analysis in plant tissues. The extraction technique for isolating phytochemicals from Brassicaceae vegetables is currently the bottleneck of the methodology. The aim of this work was to optimize this step in the analytical process. Fast and less expensive alternative, based on ultrasound-assisted extraction technique (UAE) for direct extraction of GLS into an aqueous phase and further analysis of the hydrolysis product, was optimized. Full factorial $\left(2^{k}\right)$ design followed by Central Composite Design (CCD) was used to obtain the optimum extraction conditions. Selected conditions were homogenization time $(9 \mathrm{~min})$; ultrasound bath time $(5 \mathrm{~min})$; and sample-to-solvent ratio $\left(1: 5 \mathrm{w} / \mathrm{v} \mathrm{mg} \mathrm{mL} \mathrm{m}^{-1}\right)$. The proposed analytical methodology exhibits satisfactory analytical performance in terms of linearity, precision (RSD $<2.4 \%)$, and limits of detection $\left(26 \mathrm{nmol} \mathrm{g}^{-1}\right.$ w.w.). The new analytical methodology was applied to cauliflower, cabbage, watercress, and broccoli samples with recoveries higher than $86 \%$. The UAE extraction technique was showed to be efficient for real samples analysis leading to sensible, selective, and reproducible methodology for ITC analysis.
\end{abstract}

\section{Introduction}

Glucosinolates (GLS) are the main secondary metabolites found in the Brassicaceae plants (formerly called cruciferous). To date, over than 120 different types of GLS have been described, and the common structure of all GLS comprises a $\beta$-D-thioglucose group linked to a sulfonated aldoxime moiety and a variable side chain derived from amino acids $[1,2]$. During disruption of plant material (e.g., mastication, cutting, or processing), myrosinase is released and hydrolyses GLS, forming different breakdown products, including isothiocyanates, thiocyanates, nitriles, epithionitriles, and oxazolidine-2-thiones [3]. Most of the biological activities attributed to Brassicaceae plants are related specifically to ITCs [4].
Isothiocyanates are a family of small molecules characterized by the presence of $\mathrm{a}-\mathrm{N}=\mathrm{C}=\mathrm{S}$ group and are extensively investigated for their potential to reduce the risks of chronic diseases, including atherosclerosis and cancer [5]. Epidemiological studies indicate that the human consumption of high quantities of Brassicaceae vegetables reduces the risk of developing lung, breast, prostate, colon, and rectum cancer $[6,7]$. Other biological properties include antifungal [8], antimicrobial [9], and antioxidant activities [10,11].

For those reasons, total isothiocyanates content (ITC) is considered a good indicator of bioactive compounds responsible for beneficial effects of Brassicaceae.

To obtain the ITC profile analysis, the instrumentation usually used is high performance liquid chromatography 


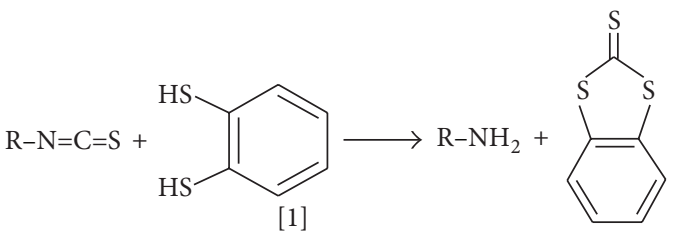

[2]

FIGURE 1: Cyclocondensation reaction of ITCs with 1,2-benzenodithiole [1], to form 1,3-benzodithiol-2-thione [2].

(HPLC) coupled to UV or evaporative light-scattering detectors [12-14] and gas chromatography coupled to mass spectrometry $[8,15,16]$.

Although there are numerous methodologies reported for quantifying ITC profile in Brassicaceae samples, in this paper we will focus on the determination of total ITC.

Reported analytical methodologies for total ITC analysis in Brassicaceae involve different sample preparation techniques along with selective and sensitive instrumentation.

Total ITC analysis is based on the cyclocondensation reaction between the carbon atom of the $-\mathrm{N}=\mathrm{C}=\mathrm{S}$ group and the thiol group of a 1,2-benzenedithiol to form 1,3-benzodithiole-2-thione (BDT) (Figure 1) and could be detected spectrophotometrically. Nevertheless, an analysis carried out by HPLC-UV was found to be the appropriate technique for ITC determination, since it allows the separation of the target analytes from interferences [17-19].

Regarding sample preparation, a solid-liquid extraction (SLE) technique has been reported using water as extractive solvent [27]. The vegetable juice is obtained by squeezing the sample with six layers of cheesecloth and hands pressure. This technique is widely used, although it is time-consuming and shows low reproducibility. Solid Phase Extraction (SPE) has also been employed for the extraction and preconcentration of these compounds [20,21]. This last method is more expensive and uses different solvents. The availability for a fast and inexpensive analytical procedures for determination of ITCs in different Brassicaceae vegetables is highly demanded for the quality control and nutraceutical research.

In this regard, the advanced extraction techniques have to demonstrate superior performance over the conventional ones. To our knowledge, there are few researches on optimized and modern extraction techniques for ITC $[22,23]$.

Ultrasound-assisted technique (UAE) is a modern alternative for the extraction of these analytes. UAE enhance the extraction procedure due to disruption of cell walls, particle-size reduction, improving the mass transfer of the cell contents as a results of cavitation bubble collapse [24]. At present, only two works have been reported using this extraction technique for glucosinolates from Indian mustard seeds (Brassica juncea L.) [25] and rocket seeds (Eruca sativa), but not for ITC.

The goal of this work was the development and validation of a rapid and less expensive analytical procedure to quantify ITCs in different matrices, using an ultrasound-assisted extraction technique (UAE), and HPLC-UV analysis. It is the first time that UAE is applied for extraction of ITCs from Brassicaceae vegetables prior HPLC-UV analysis.
A multivariate approach was used to optimize the extraction step to evaluate the effect of each selected variable and their interactions to get the highest analytical response of ITCs. The analytical performance of the proposed methodology was evaluated in terms of detection limits (LODs), repeatability and linear working range. Moreover, the procedure was applied for the determination of ITCs in different Brassicaceae matrices and its robustness was evaluated in terms of recovery factors (RF\%). Additionally the methodology was compared against that previously reported [27].

\section{Materials and Methods}

2.1. Chemicals and Reagents. 1,2-Benzenedithiol (1,2-BDT) and phenyl-ITC (PITC) stock solutions, 96 and 98\% v/v purity, respectively; were purchased from Sigma-Aldrich (Saint Luis, MO, USA) and kept at $4^{\circ} \mathrm{C}$ and light protected. Methanol $(\mathrm{MeOH})$, dichloromethane (DCM), chloroform and isopropanol, all HPLC grade, were purchased from Merck (Darmstadt, Germany). Potassium phosphate buffer $(98 \% \mathrm{~m} / \mathrm{v})$ was acquired from Biopack (Buenos Aires, Argentina). Ultrapure water $(18 \mathrm{M} \Omega \mathrm{cm})$ was obtained from a Milli-Q water purification system (Millipore, Paris, France).

\subsection{1,3-Benzodithiole-2-thione Synthesis and Characteriza-} tion. 1,3-Benzodithiole-2-thione was synthesized based on the reaction of PITC with 1,2-benzenedithiol, following a previously reported method [28]. Briefly, $5.6 \mathrm{mmol}$ of 1,2-benzenedithiol were dissolved in a solution of $12 \mathrm{~mL}$ methanol and $24 \mathrm{~mL}$ potassium phosphate buffer ( $\mathrm{pH} \mathrm{8.5)}$. PITC $(9.9 \mathrm{mmol})$ was mixed with $12 \mathrm{~mL} \mathrm{MeOH}$, and drop wise added into the 1,2-benzenedithiol solution under constant stirring. The mixture was left to react $5 \mathrm{~h}$ at room temperature under nitrogenous atmosphere and constantly stirred. The reaction product was then extracted three times with chloroform, and any residue of water in the organic phase was removed with solid sodium sulfate $\left(\mathrm{Na}_{2} \mathrm{SO}_{4}\right)$. A rotary evaporator was used to evaporate the chloroform (DecalabFbr, Buenos Aires, Argentina). The light yellow solid obtained (1,3-benzodithiole-2-thione), was purified using HPLC (conditions are described in Section 2.3.).

Structural confirmation of the synthesized compound (1,3-benzodithiole-2-thione) was conducted by three different techniques. First, the compound was injected into a gas chromatograph (GC) coupled to a flame photometric detector (Hewlett Packard 5890 Series II), using a HP5 column $30 \mathrm{~m} \times 0.30 \mathrm{~mm}, 0.25 \mu \mathrm{m}$ film thicknesses (Hewlett Packard, USA). Its retention time was compared against bibliographic data [15]. Secondly, using thin-layer chromatography (TLC, Uniplate, Silica Gel GHLF, $250 \mu \mathrm{m}$; Analtech, Inc., Newark, $\mathrm{DE})$, the retention factor coefficient of the synthesized compound was compared against reported data [29]. The spot was then dissolved with methanol and analyzed by HPLC-UV. A single peak was confirmed at $\lambda=365 \mathrm{~nm}$, resulting purity was $>99 \%$ based on total area integration (HPLC-UV conditions are described in Section 2.3.). Finally, the wavelength spectrum of the synthesized compound within the range 190$600 \mathrm{~nm}$ obtained using a Lambda $35 \mathrm{UV} / \mathrm{Vis}$ spectrometer (PerkinElmer, Shelton, CT, USA), was in agreement with data 
previously reported using the same instrumental conditions [18].

2.3. Equipment and Operating Conditions. A liquid chromatograph Konik KNK-500 series, UV/Vis detector (Konik, Barcelona, Spain) was used. HPLC column was Waters Spherisorb $\mathrm{C}_{18}$ column $(254 \times 4.6 \mathrm{~mm}$, I.D. $5 \mu \mathrm{m}$ particle size) (Milford, Massachusetts, USA). Data obtained were processed by EZ Chrom Chromatography Data System Version 6.8 software. HPLC-UV conditions were according to previous report: isocratic elution using as mobile phase $\mathrm{MeOH}$ : water $(70: 30(\mathrm{v} / \mathrm{v}))$ at $1.75 \mathrm{~mL} \mathrm{~min}^{-1} ;(\lambda=365 \mathrm{~nm})$ [27]. Peak identification was carried out by comparing retention times with reference standard. Each analytical sequence included quality control standard (PITC) and blanks to monitor background levels and possible carryover between samples.

2.4. Sampling and Sample Conditioning. All vegetable samples were purchased in local market from Mendoza, Argentina. Five fresh heads of broccoli (Brassica oleracea var. italica) were used for the optimization process, while for the recovery study five heads of cauliflower and cabbage ( $B$. oleracea var. botrytis and $B$. oleracea var. capitata, resp.) and three packages of watercress (Nasturtium officinale), recently harvested, were used. The samples were stored at $+4^{\circ} \mathrm{C}$ before performing the analysis.

2.5. Extraction Procedure and Cyclocondensation Reaction. The proposed extraction procedure was as follows: vegetable was cut in homogeneous pieces to form a single batch. A subsample was homogenized with an aliquot of water and further ultrasonicated (US-bath, $40 \mathrm{kHz}$ and $600 \mathrm{~W}$, model tb 04, TESTLAB, Buenos Aires, Argentina) in a glass beaker of $250 \mathrm{~mL}$. Hydrolysis of GLS to ITCs was carried out by stirring an aliquot of $5 \mathrm{~mL}$ homogenates for $2 \mathrm{~h}$ at $37^{\circ} \mathrm{C}$ in a water bath, conditions enough to complete the GSL hydrolysis. These variables values were selected as a consequence of previous assays $\left(37\right.$ and $20^{\circ} \mathrm{C}$, for 2 and $8 \mathrm{~h}$. After $2 \mathrm{~h}$, no significant difference was obtained (data not shown)).

After extraction, the cyclocondensation was carried out [30]: briefly, an aliquot of $250 \mu \mathrm{L}$ of hydrolyzed homogenate was mixed with $250 \mu \mathrm{L} 100 \mathrm{mmol} \mathrm{L}^{-1}$ potassium phosphate buffer (pH 8.5) and $500 \mu \mathrm{L} 10 \mathrm{mmol} \mathrm{L}^{-1}$ 1,2-benzenedithiol in 2-propanol in a $4 \mathrm{~mL}$ vial and kept at $65^{\circ} \mathrm{C}, 2 \mathrm{~h}$. Then, mixture was centrifuged (Eppendorf MiniSpin plus, Hamburg, Germany), at $14000 \mathrm{rpm}$ (15339 g) for $5 \mathrm{~min}$ and further filtered with a $0.22 \mu \mathrm{m}$ filter membrane before injection into the HPLC system.

2.6. Experimental Design and Statistical Analysis. Multifactorial experimental designs were carried out. Initially, a full factorial experimental design $\left(2^{k}\right)$ was used to evaluate the significance of experimental variables, as well as their interaction on the analytical response. After multiple linear regression and analysis of variance, a Pareto chart was used to select those variables that significantly influenced the extraction. Selected variables were then optimized using a Central Composite Design (CCD) to obtain the highest analytical response. This latter study allowed identifying those conditions to obtain the maximum analytical response of ITCs. Five replicates at the center point of design were used to estimate the error. Experiments were conducted randomly. ITCs chromatographic data was taken as the analytical response for the combination of the independent variables.

Data was analyzed by Design Expert 8.0.7.1. The statistical differences among the values obtained under experimental conditions were established by ANOVA and Tukey test ( $p=$ 0.05) using InfoStat software, 2013.

2.7. Comparative Method. The optimized extraction technique was compared against the methodology proposed by Jiao et al. [27]. A broccoli single batch was analyzed in triplicate using the proposed and reference methodologies. Briefly, fresh broccoli sample $(150 \mathrm{~g})$ was mixed $(1 \mathrm{~min})$ with $100 \mathrm{~mL}$ deionized water to obtain a vegetable paste. The paste was then wrapped with six layers of cheesecloth and squeezed to release the juice into a beaker. The process was repeated twice with the addition of $125 \mathrm{~mL}$ deionized water each time. The three extracts were then combined, and the volume was flushed to $500 \mathrm{~mL}$ with deionized water. Ten milliliters of the final vegetable juice was filtered under vacuum to obtain a clear filtrate. ITCs were analyzed by HPLC after cyclocondensation reaction. The statistical differences between the values obtained under the two extraction were established by undertaking ANOVA and Tukey test $(p=$ 0.05) using InfoStat software, 2013.

\section{Results}

3.1. Screening Design. Table 1 summarizes the conditions established by the multifactorial experimental design for every run. In Figure 2, it is possible to observe the Pareto chart obtained after data analysis. Based on this, ultrasound bath time (referred to as $C$ in the figure) and homogenization time (referred to as $B$ in the figure) are the two variables that significantly influence the analytical response, showing a positive influence on ITCs extraction $(p \leq 0.05)$. Sampleto-solvent ratio ( $A$ in Figure 2$)$ and salting out effect $(D$ in Figure 2) did not significantly influence the analytical response.

3.2. Central Composite Design and Surface Plot. The influential variables were optimized using a Central Composite Design. Table 2 shows the range of independent variables and their levels, expressing the ITCs concentrations $\left(\mathrm{nmolg}^{-1}\right)$ in fresh broccoli obtained after each run. The resulting behavior of the analytical response of the ITCs under the influence of the studied variables was best explained by the use of a quadratic model (1), where homogenization time is represented by $X_{m}$ and ultrasound bath time by $X_{n}$ :

$$
\begin{aligned}
{[Y]=} & -346.7+18.3 X_{n}+173.2 X_{m}-1.73 X_{n}{ }^{2} \\
& -9.7 X_{m}{ }^{2} .
\end{aligned}
$$


TABle 1: Multifactorial experimental design $2^{4}$ for total ITCs extraction.

(a)

\begin{tabular}{lcc}
\hline \multirow{2}{*}{ Evaluated variables } & \multicolumn{2}{c}{ Levels } \\
& Low & High \\
\hline (A) Sample-to-solvent ratio (w/v) & $1: 5$ & $3: 5$ \\
(B) Homogenization time $(\mathrm{min})$ & 2 & 7 \\
(C) Use of ultrasound bath $(\mathrm{min})$ & 0 & 5 \\
(D) Salting out effect $\left(\mathrm{mg} \mathrm{mL}^{-1} \mathrm{NaCl}\right)$ & 0 & 50 \\
\hline
\end{tabular}

(b)

\begin{tabular}{lcccc}
\hline \multirow{2}{*}{ Run } & \multicolumn{4}{c}{ Extraction condition } \\
& $A$ & $B$ & $C$ & $D$ \\
\hline 1 & $1: 5$ & 5 & 5 & 0 \\
2 & $3: 5$ & 2 & 0 & 50 \\
3 & $1: 5$ & 2 & 0 & 50 \\
4 & $1: 5$ & 5 & 0 & 50 \\
5 & $3: 5$ & 5 & 0 & 50 \\
6 & $3: 5$ & 5 & 5 & 50 \\
7 & $3: 5$ & 2 & 5 & 50 \\
8 & $3: 5$ & 5 & 5 & 0 \\
9 & $3: 5$ & 2 & 0 & 0 \\
10 & $1: 5$ & 5 & 5 & 50 \\
11 & $1: 5$ & 2 & 0 & 0 \\
12 & $1: 5$ & 5 & 0 & 0 \\
13 & $3: 5$ & 2 & 5 & 0 \\
14 & $1: 5$ & 5 & 0 & 0 \\
15 & $1: 5$ & 2 & 5 & 50 \\
16 & $1: 5$ & 2 & 5 & 0 \\
\hline
\end{tabular}

The predictive ability of the obtained experimental model shows, through the comparison between the experimental values $\left(443.7 \pm 13.4 \mathrm{nmolg}^{-1}\right.$ w.w. $)$ and predicted values $\left(455.9 \pm 0.4 \mathrm{nmol} \mathrm{g}^{-1}\right.$ w.w. $)$, no significant differences $(p \geq$ $0.05)$ for an identical combination of variables.

3.3. Analytical Performance and Comparison. Different concentrations of the 1,3-benzodithiole-2-thione solution were added to fresh broccoli samples to obtain the calibration curve. The proposed methodology showed satisfactory linearity within the concentration range of 50-2000 (nmol g ${ }^{-1}$ w.w.) total ITCs in fresh broccoli inflorescences $\left(r^{2}=0.996\right)$. The detection limit (LOD) calculated as three times signal-to-noise ratio $(\mathrm{S} / \mathrm{N}=3)$ was $26 \mathrm{nM}$. The precision of the extraction method was evaluated over five replicates at $100 \mathrm{nmol} \mathrm{g}^{-1}$ w.w., leading to a Relative Standard Deviation (RSD) of 2.4\% (95\% confidence interval). To evaluate accuracy of the analytical methodology, a recovery study of ITCs at two different concentrations (300 and $400 \mathrm{nmol} \mathrm{g}^{-1}$ w.w.) was carried out over different Brassicaceae vegetables samples (cauliflower, cabbage, and watercress). As seen in Table 3, satisfactory recoveries, in the range of $86-96 \%$, were obtained. The best recovery was
TABLE 2: Analytical responses values obtained for ITCs concentration for the Central Composite Design arrangement. Analytical responses and predicted values for isothiocyanate production (nmol g ${ }^{-1}$ w.w.).

(a)

\begin{tabular}{lccccc}
\hline \multirow{2}{*}{ Independent variables } & \multicolumn{5}{c}{ Levels } \\
& -2 & -1 & 0 & 1 & 2 \\
\hline$X_{n}$ : ultrasound time $(\min )$ & 0.34 & 2.00 & 6.00 & 10.00 & 11.66 \\
$X_{m}$ : homogenization time $(\min )$ & 3.96 & 5.00 & 7.50 & 10.00 & 11.04 \\
\hline
\end{tabular}

(b)

\begin{tabular}{lcccc}
\hline \multirow{2}{*}{ Run } & $X_{n}$ & $X_{m}$ & \multicolumn{2}{c}{ ITCs production $\left(\mathrm{nmol} \mathrm{g}^{-1}\right.$ w.w.) } \\
& & Observed values & Predicted values \\
\hline 1 & -1 & -1 & 252.6 & 307.2 \\
2 & 2 & 0 & 406.0 & 386.2 \\
3 & -1 & 1 & 447.2 & 447.5 \\
4 & 1 & -1 & 276.2 & 286.9 \\
5 & 0 & 0 & 449.6 & 455.4 \\
6 & 0 & -2 & 273.6 & 234.7 \\
7 & 0 & 2 & 446.2 & 433.1 \\
8 & -2 & 0 & 444.7 & 414.9 \\
9 & 0 & 0 & 434.3 & 455.4 \\
10 & 1 & 1 & 399.5 & 427.2 \\
11 & 0 & 0 & 453.5 & 455.4 \\
12 & 0 & 0 & 469.6 & 455.4 \\
13 & 0 & 0 & 475.6 & 455.4 \\
\hline
\end{tabular}

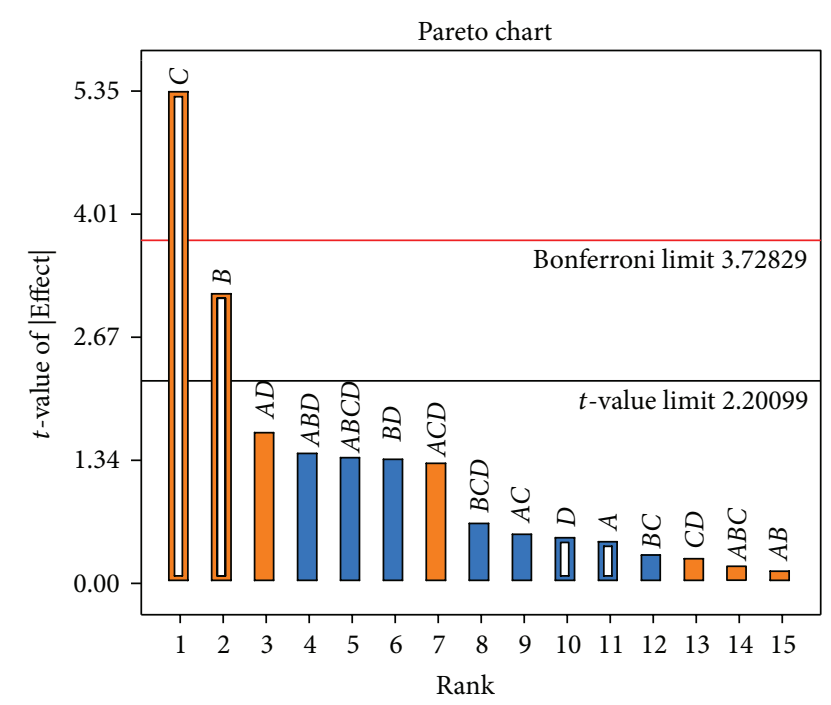

FIGURE 2: Pareto chart obtained after screening design. Variables: (A) Sample-to-solvent ratio $\left(1: 5\right.$ and $\left.3: 5 \mathrm{w} / \mathrm{v}\left(\mathrm{mg} \mathrm{mL}^{-1}\right)\right) ;(B)$ homogenization time (2 and $7 \mathrm{~min}$ ); $(C)$ use of ultrasound bath $(0$ and $5 \mathrm{~min})$; and $(D)$ salting out effect $\left(0\right.$ and $\left.50 \mathrm{mg} \mathrm{mL}^{-1} \mathrm{NaCl}\right)$.

obtained for cabbage (92-96\%). Broccoli and cauliflower show similar results (90-95\%), while the lowest was obtained for watercress (86-93\%). In Figure 4, it is possible to observe the results of comparative study between both extraction 
TABLE 3: Total ITCs concentration found in Brassicaceae samples (95\% confidence interval, $n=3$ ).

\begin{tabular}{|c|c|c|c|c|}
\hline Sample & Basal level $\left(\mathrm{nmol} \mathrm{g}^{-1}\right)$ & Added level $\left(\mathrm{nmolg}^{-1}\right)$ & Found level $\left(\mathrm{nmol} \mathrm{g}^{-1}\right)$ & Recovery $(\%)^{\mathrm{a}}$ \\
\hline & & - & & - \\
\hline \multirow[t]{3}{*}{ Broccoli } & $221 \pm 4.0$ & 300 & $491 \pm 5.31$ & 90 \\
\hline & & 400 & $601 \pm 14.8$ & 95 \\
\hline & & - & & - \\
\hline \multirow[t]{3}{*}{ Cauliflower } & $145 \pm 5.0$ & 300 & $415 \pm 26.6$ & 90 \\
\hline & & 400 & $526 \pm 20.7$ & 95 \\
\hline & & - & & - \\
\hline \multirow[t]{3}{*}{ Cabbage } & $89 \pm 4.8$ & 300 & $364 \pm 14.6$ & 92 \\
\hline & & 400 & $474 \pm 22.2$ & 96 \\
\hline & & - & & - \\
\hline \multirow[t]{2}{*}{ Watercress } & $153 \pm 12.6$ & 300 & $411 \pm 16.1$ & 86 \\
\hline & & 400 & $524 \pm 59.4$ & 93 \\
\hline
\end{tabular}

${ }^{\mathrm{a}}[($ Found - basal $) /$ added $] \times 100$.

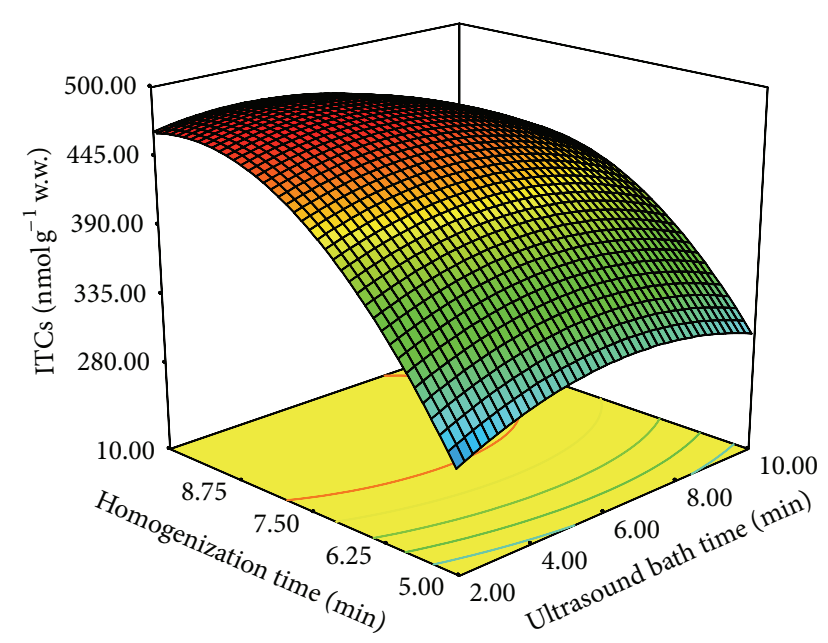

FIGURE 3: Effect of homogenization and ultrasound bath times on total ITCs extraction from broccoli samples.

techniques, the optimized against the proposed by Jiao et al. [27]. The use of the proposed technique leads to an average concentration of $443.7 \pm 13.4$ nmol ITCs $g^{-1}$ w.w., while the second was $187.1 \pm 11.8$ nmol ITCs g ${ }^{-1}$ w.w.

Representative chromatograms of vegetables extracts by UAE-HPLC-UV analysis are shown in Figure 5. Additionally, the studied ITCs did not show a significant shift in the retention time and/or sensitivity in the chromatographic analysis.

\section{Discussion}

Using the screening design, 4 variables with 2 levels each were evaluated on $10 \mathrm{~g}$ sample, including sample-to-solvent ratio $(1: 5$ and $3: 5 \mathrm{w} / \mathrm{v})$, use of ultrasound bath ( 0 and $5 \mathrm{~min})$, homogenization time ( 2 and $7 \mathrm{~min})$, and salting out effect ( 0 and $50 \mathrm{mg} \mathrm{mL}^{-1} \mathrm{NaCl}$ ). The results proved that the use of ultrasound bath and homogenization time significantly

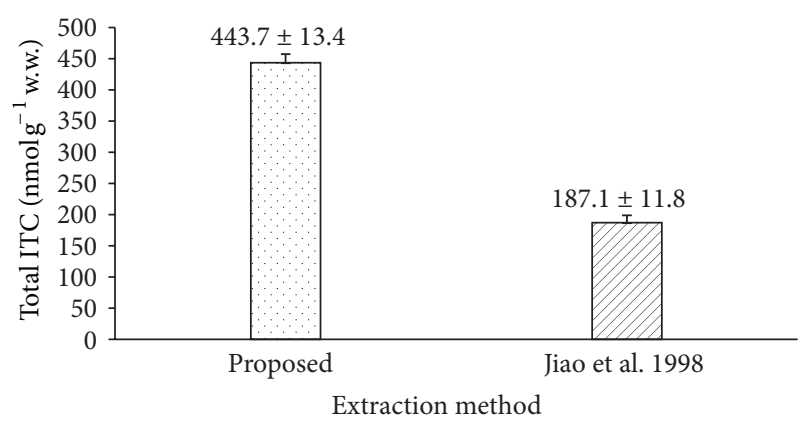

FIGURE 4: Comparison of different extraction techniques (proposed and reported by Jiao et al. [27]).

influence the extraction of ITCs; therefore, both variables were selected for further optimization.

The objective in the optimization process was to find a combination of homogenization time and ultrasound bath time that lead to the best analytical response. Experimental values of ITCs extraction were analyzed by multiple regressions to fit the second-order regression equation. CCD was carried out, evaluating the combination of two levels each in 13 runs. Statistical parameters corresponding to the fitting for resolution demonstrated the significance of the models $(p<0.05)$. The obtained surface plot (Figure 3 ) was used to select the combination of optimal variables values for maximum response of ITCs. The best ITCs analytical response was obtained with a 5 min ultrasound bath time and a 9 min homogenization time. The significant difference $(p>0.05)$ of the comparative study (Figure 4$)$ demonstrates an improvement of the efficiency extraction two times higher. This result was based on the ultrasonically induced cavitation. Mechanical effects as microfractures and disruption of cell walls increase the permeability of the plant tissues facilitating the release of their contents, in contrast to conventional maceration. These effects were observed under scanning electron microscopy [31].

Besides these results, the proposed technique was simpler and involved minimum sample manipulation leading to a 
TABLE 4: Comparison of UAE-HPLC-UV with other analytical methods previously reported for total ITCs determination employing the cyclocondensation reaction.

\begin{tabular}{|c|c|c|c|c|c|c|}
\hline Method & $\begin{array}{c}\text { Bioactive compounds } \\
\text { analysed }\end{array}$ & Sample & LOD & RSD \% & Recovery (\%) & Reference \\
\hline SPE-HPLC-DAD & Total isothiocyanate & Human plasma & $30 \mathrm{nM}$ & & $>93.3$ & Ye et al. 2002 [21] \\
\hline LLE-HPLC-UV & Total isothiocyanate & Human plasma & $20 \mathrm{nM}$ & $<6.5$ & $>96.6$ & Liebes et al. 2001 [26] \\
\hline Boiling water-GC-MS & Total isothiocyanate & $\begin{array}{l}\text { Chinese medicinal herbs and } \\
\text { cruciferous vegetables }\end{array}$ & $35 \mathrm{nM}$ & & $>90.0$ & Choi et al. 2004 [15] \\
\hline SPE-HPLC-UV & Total isothiocyanate & Broccoli & $25 \mu \mathrm{mol}$ & & $>91.2$ & Bertelli et al. 1998 [20] \\
\hline UAE-HPLC-UV & Total isothiocyanate & $\begin{array}{l}\text { Broccoli, cauliflower, watercress, } \\
\text { and cabbage }\end{array}$ & $26 \mathrm{nM}$ & $<2.4$ & $>86.0$ & Proposed work \\
\hline
\end{tabular}

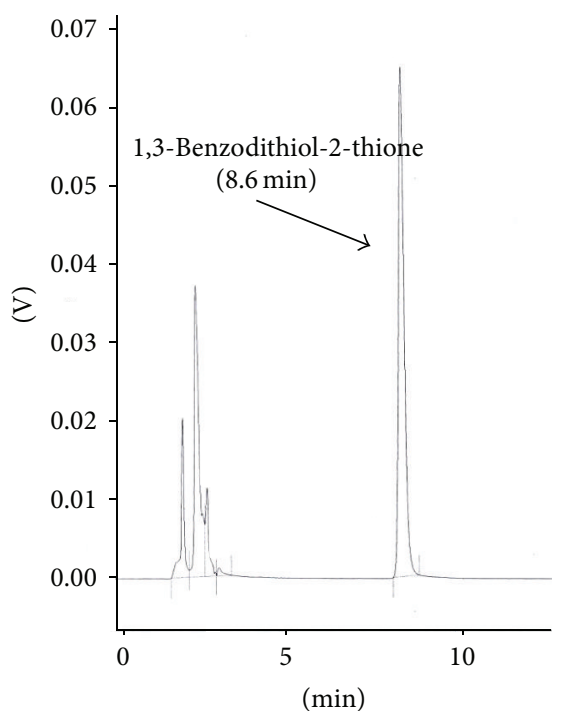

(a)

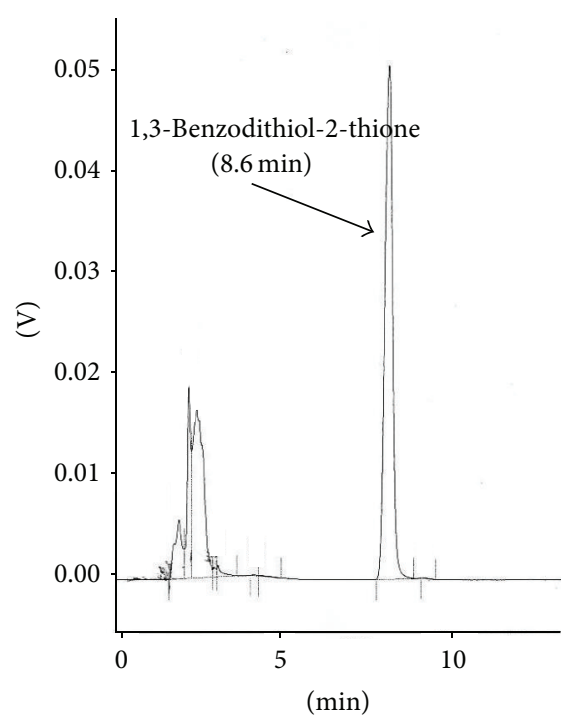

(c)

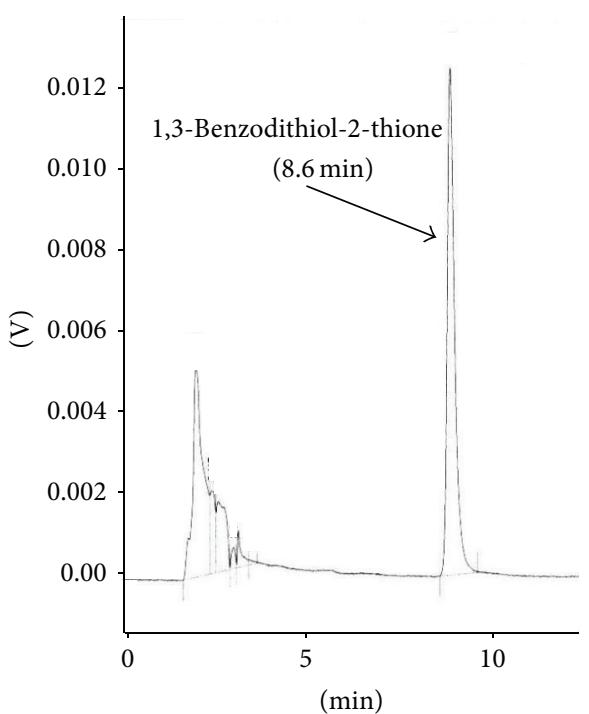

(b)

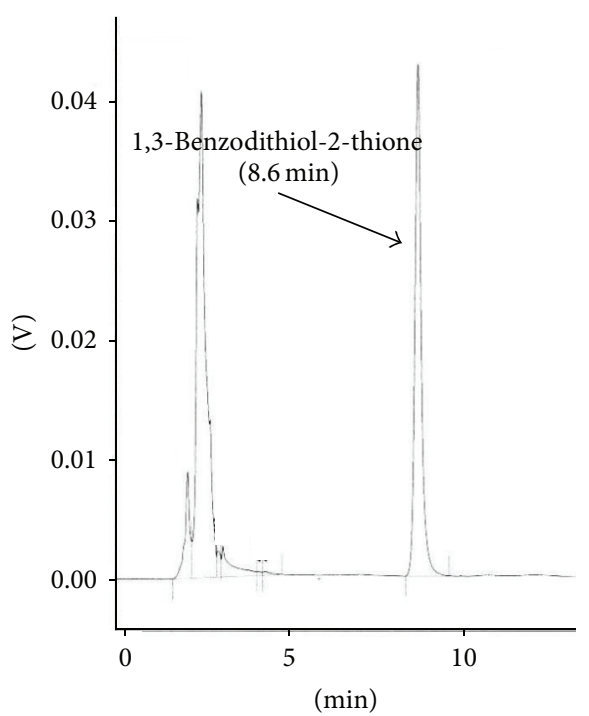

(d)

FIGURE 5: Chromatograms of vegetables extracts after the UAE-HPLC-UV analysis. Broccoli (a), cabbage (b), watercress (c) and cauliflower (d). Characteristic peak of 1,3-benzodithiole-2-thione is shown at $8.6 \mathrm{~min}$. 
higher throughput sample analysis, positively affecting the analytical performance of the whole methodology and thus its sensibility.

In Table 4 , it is possible to observe that the analytical performance of UAE-HPLC-UV for ITC determination in different matrices samples was compared with other previously reported analytical methodologies. It was observed that the LODs data obtained with the methodology that employed boiling water-GC-MS [15] is less sensitive than ours. The methodologies obtained both by LLE-HPLC-UV [26] and SPE-HPLC-DAD [21] have shown comparable LODs with the proposed methodology, but their results were obtained from human plasma matrices. The mean RSDs values were not comparable because the only data available are from different matrices.

Regarding accuracy values obtained, these are in the order reported by other authors (as shown in Figure 4). In view of the informed results, the proposed methodology showed low consumption of solvent, high recoveries, short extraction time, low matrix interference, and good merit figures compared to other methods, which are comparative advantages the proposed methodology. Therefore, this methodology contributes to a sustainable environment by the limited use of organic solvents and, in addition, represents a simple, rapid, and less expensive extraction technique.

\section{Conclusion}

The particular factors of a real matrix, as well as all operative variables that condition the analytical responses of the analytes, were evaluated. The use of a multivariate statistical approach all along the process is appropriate for characterizing and optimizing the analytical methodology that has been proposed. This analytical methodology can be successfully applied in routine analysis to determine wide range levels of total ITCs in different Brassicaceae species.

\section{Conflict of Interests}

The authors declare that there is no conflict of interests regarding the publication of this paper.

\section{Acknowledgments}

This work was supported by Consejo Nacional de Investigaciones Científicas y Técnicas (CONICET), Universidad Nacional de Cuyo, Agencia Nacional de Promoción Científica y Técnica, and Organization for Prohibition of Chemical Weapons.

\section{References}

[1] A. Crozier, M. N. Clifford, and H. Ashihara, Plant Secondary Metabolites Occurrence, Structure and Role in the Human Diet, Blackwell, Oxford, UK, 1st edition, 2006.

[2] B. A. Halkier and J. Gershenzon, "Biology and biochemistry of glucosinolates," Annual Review of Plant Biology, vol. 57, pp. 303$333,2006$.
[3] A. M. Bones and J. T. Rossiter, "The enzymic and chemically induced decomposition of glucosinolates," Phytochemistry, vol. 67, no. 11, pp. 1053-1067, 2006.

[4] G. R. De Nicola, M. Bagatta, E. Pagnotta et al., "Comparison of bioactive phytochemical content and release of isothiocyanates in selected brassica sprouts," Food Chemistry, vol. 141, no. 1, pp. 297-303, 2013.

[5] A. T. Dinkova-Kostova and R. V. Kostov, "Glucosinolates and isothiocyanates in health and disease," Trends in Molecular Medicine, vol. 18, no. 6, pp. 337-347, 2012.

[6] L. R. Ferguson and R. C. Schlothauer, "The potential role of nutritional genomics tools in validating high health foods for cancer control: broccoli as example," Molecular Nutrition \& Food Research, vol. 56, no. 1, pp. 126-146, 2012.

[7] J. V. Higdon, B. Delage, D. E. Williams, and R. H. Dashwood, "Cruciferous vegetables and human cancer risk: epidemiologic evidence and mechanistic basis," Pharmacological Research, vol. 55, no. 3, pp. 224-236, 2007.

[8] R. Troncoso, C. Espinoza, A. Sánchez-Estrada, M. E. Tiznado, and H. S. García, "Analysis of the isothiocyanates present in cabbage leaves extract and their potential application to control Alternaria rot in bell peppers," Food Research International, vol. 38, no. 6, pp. 701-708, 2005.

[9] S.-H. Hu, J.-C. Wang, H.-F. Kung, J.-T. Wang, W.-L. Lee, and Y.-H. Yang, "Antimicrobial effect of extracts of cruciferous vegetables," Kaohsiung Journal of Medical Sciences, vol. 20, no. 12, pp. 591-599, 2004.

[10] N. Hounsome, B. Hounsome, D. Tomos, and G. Edwards-Jones, "Changes in antioxidant compounds in white cabbage during winter storage," Postharvest Biology and Technology, vol. 52, no. 2, pp. 173-179, 2009.

[11] M.-A. Lee, J.-H. Choi, Y.-S. Choi et al., "The antioxidative properties of mustard leaf (Brassica juncea) kimchi extracts on refrigerated raw ground pork meat against lipid oxidation," Meat Science, vol. 84, no. 3, pp. 498-504, 2010.

[12] H. Liang, Q. P. Yuan, H. R. Dong, and Y. M. Liu, “Determination of sulforaphane in broccoli and cabbage by high-performance liquid chromatography," Journal of Food Composition and Analysis, vol. 19, no. 5, pp. 473-476, 2006.

[13] K. Nakagawa, T. Umeda, O. Higuchi, T. Tsuzuki, T. Suzuki, and T. Miyazawa, "Evaporative light-scattering analysis of sulforaphane in broccoli samples: quality of broccoli products regarding sulforaphane contents," Journal of Agricultural and Food Chemistry, vol. 54, no. 7, pp. 2479-2483, 2006.

[14] L. Song, J. J. Morrison, N. P. Botting, and P. J. Thornalley, "Analysis of glucosinolates, isothiocyanates, and amine degradation products in vegetable extracts and blood plasma by LCMS/MS," Analytical Biochemistry, vol. 347, no. 2, pp. 234-243, 2005.

[15] M. M. F. Choi, S. Shuang, H. Y. Lai et al., "Gas chromatographymass spectrometric determination of total isothiocyanates in Chinese medicinal herbs," Analytica Chimica Acta, vol. 516, no. 1-2, pp. 155-163, 2004.

[16] D. Zhao, J. Tang, and X. Ding, "Analysis of volatile components during potherb mustard (Brassica juncea, Coss.) pickle fermentation using SPME-GC-MS," LWT-Food Science and Technology, vol. 40, no. 3, pp. 439-447, 2007.

[17] Y. Zhang, "The 1,2-benzenedithiole-based cyclocondensation assay: a valuable tool for the measurement of chemopreventive isothiocyanates," Critical Reviews in Food Science and Nutrition, vol. 52, no. 6, pp. 525-532, 2012. 
[18] Y. Zhang, C.-G. Cho, G. H. Posner, and P. Talalay, "Spectroscopic quantitation of organic isothiocyanates by cyclocondensation with vicinal dithiols," Analytical Biochemistry, vol. 205, no. 1, pp. 100-107, 1992.

[19] Y. Zhang, K. L. Wade, T. Prestera, and P. Talalay, "Quantitative determination of isothiocyanates, dithiocarbamates, carbon disulfide, and related thiocarbonyl compounds by cyclocondensation with 1,2-benzenedithiol," Analytical Biochemistry, vol. 239, no. 2, pp. 160-167, 1996.

[20] D. Bertelli, M. Plessi, D. Braghiroli, and A. Monzani, "Separation by solid phase extraction and quantification by reverse phase HPLC of sulforaphane in broccoli," Food Chemistry, vol. 63, no. 3, pp. 417-421, 1998.

[21] L. Ye, A. T. Dinkova-Kostova, K. L. Wade, Y. Zhang, T. A. Shapiro, and P. Talalay, "Quantitative determination of dithiocarbamates in human plasma, serum, erythrocytes and urine: pharmacokinetics of broccoli sprout isothiocyanates in humans," Clinica Chimica Acta, vol. 316, no. 1-2, pp. 43-53, 2002.

[22] A. M. Ares, J. Bernal, M. T. Martín, J. L. Bernal, and M. J. Nozal, "Optimized formation, extraction, and determination of sulforaphane in broccoli by liquid chromatography with diode array detection," Food Analytical Methods, vol. 7, no. 3, pp. 730740, 2014.

[23] Q. Deng, K. G. Zinoviadou, C. M. Galanakis et al., "The effects of conventional and non-conventional processing on glucosinolates and its derived forms, isothiocyanates: extraction, degradation, and applications," Food Engineering Reviews, vol. 7, no. 3, pp. 357-381, 2015.

[24] M. I. Soares Melecchi, V. Flores Péres, C. Dariva et al., "Optimization of the sonication extraction method of Hibiscus tiliaceus L. flowers," Ultrasonics Sonochemistry, vol. 13, no. 3, pp. 242-250, 2006.

[25] T. Wang, H. Liang, and Q. Yuan, "Optimization of ultrasonicstimulated solvent extraction of sinigrin from Indian mustard seed (Brassica Juncea L.) using response surface methodology," Phytochemical Analysis, vol. 22, no. 3, pp. 205-213, 2011.

[26] L. Liebes, C. C. Conaway, H. Hochster et al., "High-performance liquid chromatography-based determination of total isothiocyanate levels in human plasma: application to studies with 2phenethyl isothiocyanate," Analytical Biochemistry, vol. 291, no. 2, pp. 279-289, 2001.

[27] D. Jiao, M. C. Yu, J. H. Hankin, S.-H. Low, and F.-L. Chung, "Total isothiocyanate contents in cooked vegetables frequently consumed in Singapore," Journal of Agricultural and Food Chemistry, vol. 46, no. 3, pp. 1055-1058, 1998.

[28] M. Kristensen, K. S. Krogholm, H. Frederiksen et al., "Improved synthesis methods of standards used for quantitative determination of total isothiocyanates from broccoli in human urine," Journal of Chromatography B, vol. 852, no. 1-2, pp. 229-234, 2007.

[29] F.-L. Chung, D. Jiao, S. M. Getahun, and M. C. Yu, "A urinary biomarker for uptake of dietary isothiocyanates in humans," Cancer Epidemiology Biomarkers \& Prevention, vol. 7, no. 2, pp. 103-108, 1998.

[30] L. Tang, J. D. Paonessa, Y. Zhang, C. B. Ambrosone, and S. E. McCann, "Total isothiocyanate yield from raw cruciferous vegetables commonly consumed in the United States," Journal of Functional Foods, vol. 5, no. 4, pp. 1996-2001, 2013.

[31] M. Vinatoru, "An overview of the ultrasonically assisted extraction of bioactive principles from herbs," Ultrasonics Sonochemistry, vol. 8, no. 3, pp. 303-313, 2001. 

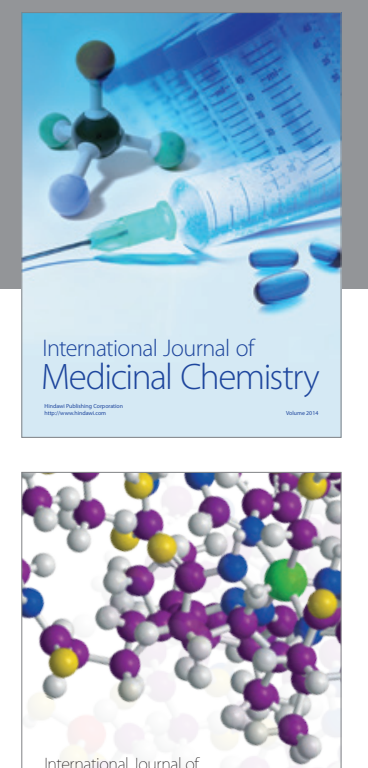

\section{Carbohydrate} Chemistry

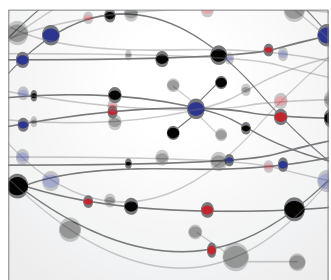

The Scientific World Journal
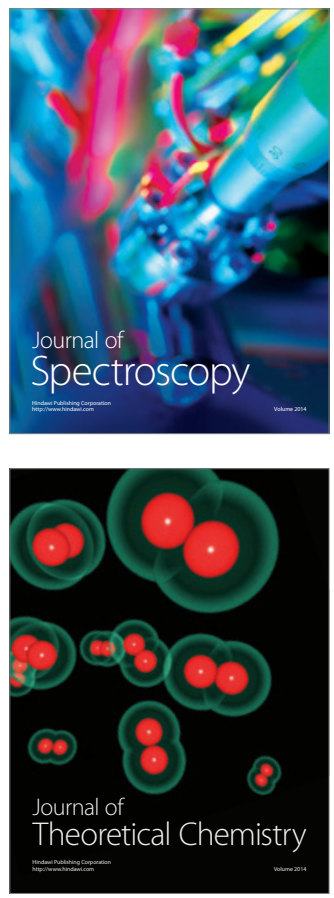
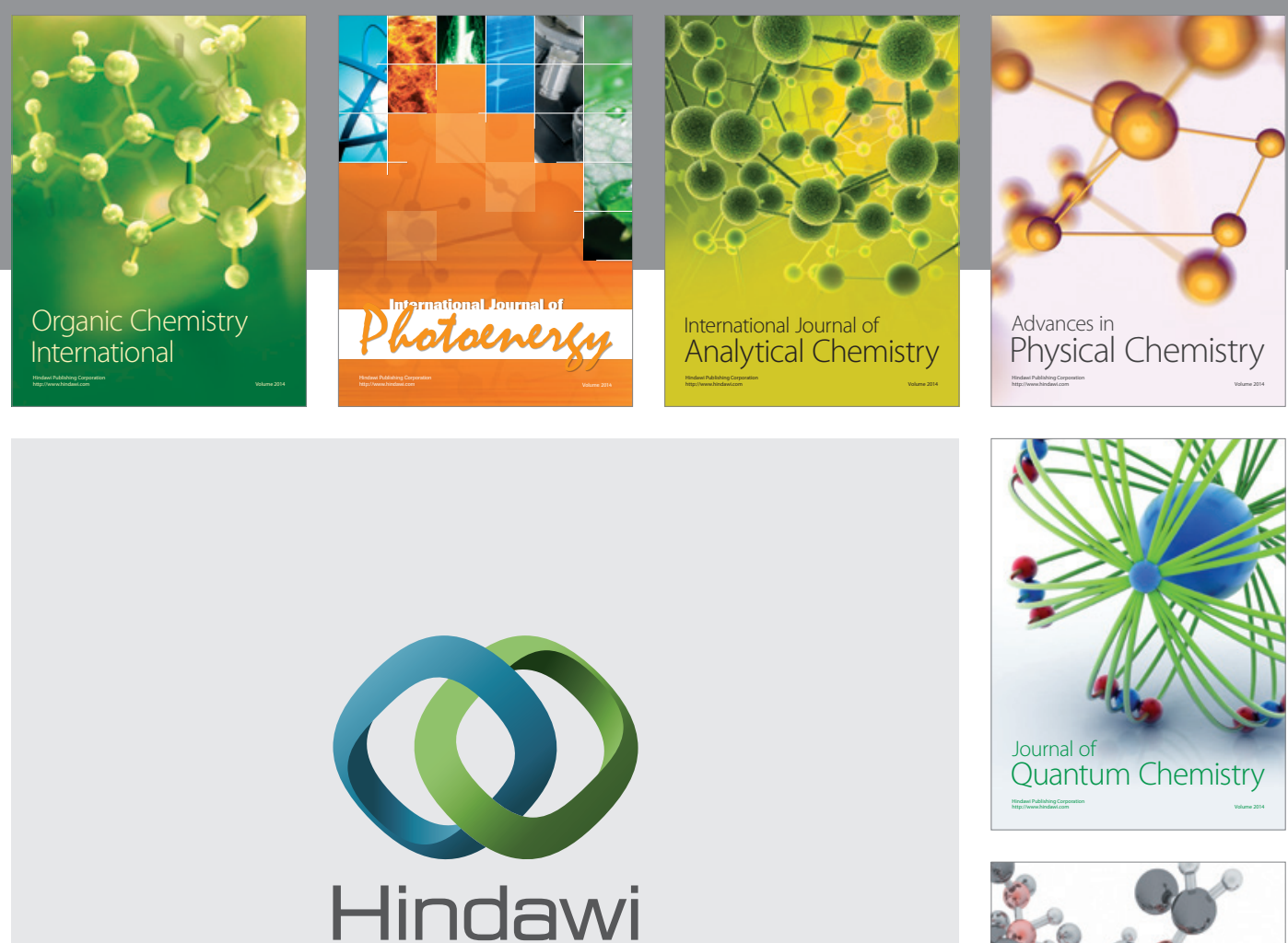

Submit your manuscripts at

http://www.hindawi.com

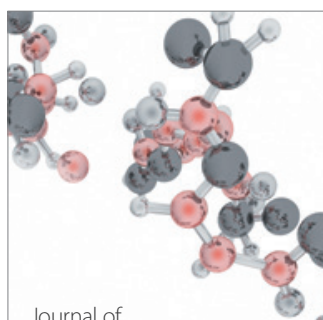

Analytical Methods

in Chemistry

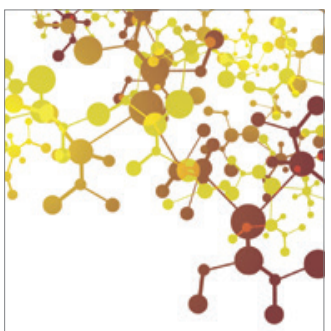

Journal of

Applied Chemistry

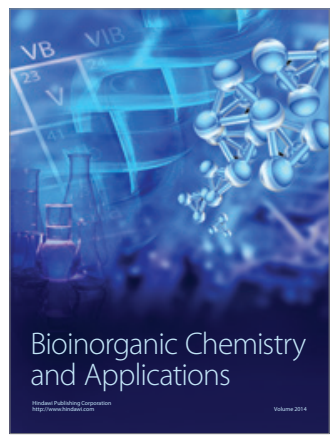

Inorganic Chemistry
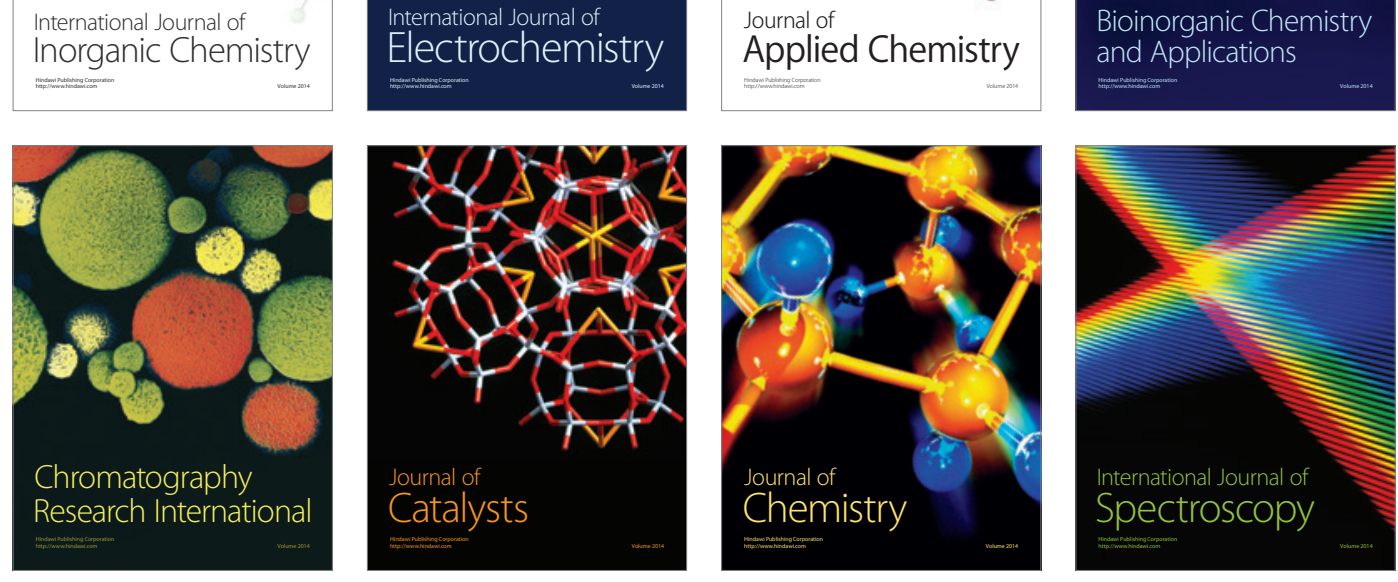